\title{
Chromosomal composition of the genome in the monomorphic diplokaryotic microsporidium Paranosema grylli: analysis by two-dimensional pulsed-field gel electrophoresis
}

\author{
Elena Nassonova ${ }^{1}$, Emmanuel Cornillot $^{2}$, Guy Méténier $^{2}$, Nina Agafonova ${ }^{1}$, Boris Kudryavtsev $^{1}$, \\ Sergei Skarlato ${ }^{1}$ and Christian P. Vivarès ${ }^{2}$ \\ ${ }^{1}$ Institute of Cytology, Russian Academy of Sciences, Tikhoretsky Ave. 4, 194064 St. Petersburg, Russia; \\ ${ }^{2}$ Equipe de Parasitologie Moléculaire et Cellulaire, UMR CNRS 6023, Université Blaise Pascal, 63177-Aubière cedex, France
}

Key words: Microsporidia, diplokaryon, molecular karyotype, KARD 2-D PFGE, chromosome-length polymorphism, ploidy, meiosis

\begin{abstract}
The molecular karyotype of Paranosema grylli Sokolova, Seleznev, Dolgikh et Issi, 1994, a monomorphic diplokaryotic microsporidium, comprises numerous bright and faint bands of nonstoichiometric staining intensity. Restriction analysis of chromosomal DNAs by "karyotype and restriction display" 2-D PFGE has demonstrated that the complexity of molecular karyotype of $P$. grylli is related to the pronounced length polymorphism of homologous chromosomes. The background of this phenomenon is discussed in the context of ploidy state, reproductive strategy and population structure in this microsporidium. We propose that the remarkable size variation between homologous chromosomes in P. grylli may be a consequence of ectopic recombination at the chromosome extremities.
\end{abstract}

More than half of microsporidian genera possess a diplokaryotic nuclear apparatus which consists of two morphologically identical adjacent nuclei that divide synchronously and equally. In the life cycle many diplokaryotic microsporidia display alternation of a diplokaryotic and a monokaryotic nuclear arrangement, accompanied with respective ploidy changes. However, some diplokaryotic microsporidia show no nuclear alternation. They are usually recognized as "monomorphic diplokaryotic microsporidia" because their life cycle is thought to be entirely diplokaryotic, involving only one sporogony sequence which results in the formation of diplokaryotic spores. This non-phylogenetic assemblage includes Nosema, the most diverse genus of the phylum Microsporidia, and about 20 other genera. Neither karyogamy nor diplokaryon dissociation has been clearly demonstrated in these organisms, and so far they are believed to be asexual. It is generally supposed that the paired nuclei of their diplokarya never recombine and therefore evolve independently. The genetic consequences of presumably permanent diplokaryosis remain unclear.

The genome organisation in monomorphic diplokaryotic microsporidia is poorly understood. The overall composition of their genome, in particular the total number of chromosomes as well as the chromosome content and ploidy level of each nucleus of the diplokaryon, is unknown. The small size of chromosomes, their loose condensation in mitosis and the absence of meiosis in the life cycle of monomorphic diplokaryotic microsporidia hinder direct light-microscopic analysis of their karyotypes. Knowledge of the chromosomal composition in these microsporidia resulted from molecular karyotyping based on pulsed-field gel electrophoresis (PFGE) (Méténier and Vivarès 2001). Molecular karyotypes were reported for few Nosema spp. and Paranosema spp. (Munderloh et al. 1990, Malone and McIvor 1993, Kawakami et al. 1994, Streett 1994, Nassonova et al. 1998). These studies provided information on the number of chromosomal bands observed in PFGE gels and the size of resolved chromosomal DNAs. However, the exact number of chromosomes could not be determined with any confidence based solely on PFGE data. No attempts have been made so far to identify the individual chromosomes in the molecular karyotypes of these microsporidia.

In this paper we discuss the results of extended analysis of the karyotype in the monomorphic diplokaryotic microsporidium Paranosema grylli Sokolova, Seleznev, Dolgikh et Issi, 1994, a fat body parasite of the cricket Gryllus bimaculatus Deg. We performed molecular karyotyping of this species and attempted to further characterise the resolved chromosomal DNAs by specific patterns of the restriction fragments. We applied an efficient protocol of chromosomal DNA digestion and in-gel radioactive labeling coupled with twodimensional PFGE, which was termed "karyotype and restriction display" 2-D PFGE (KARD 2-D PFGE)

This paper was presented at the NATO Advanced Research Workshop "Emergent Pathogens in the 21st Century: First United Workshop on Microsporidia from Invertebrate and Vertebrate Hosts", held in České Budějovice, Czech Republic, July 12-15, 2004. 
(Brugère et al. 2000a, 2001). This technique was successfully applied to chromosome identification in the genome of the microsporidium Encephalitozoon cuniculi (Brugère et al. 2000b). KARD 2-D PFGE analysis of the genome of $P$. grylli revealed a complex chromosomal composition in this organism related to a pronounced chromosome-length polymorphism. Identification of individual chromosomes permitted estimation of the number of putative linkage groups and the haploid genome size in $P$. grylli. We discuss the possible background of chromosomal polymorphism in relation to the nuclear organisation, reproductive strategy, and population structure in this monomorphic diplokaryotic microsporidium. We propose that the chromosome-length polymorphism in $P$. grylli can be a consequence of ectopic recombination at the chromosome extremities.

\section{MATERIALS AND METHODS}

Host and parasite cultures. Culture of the cricket Gryllus bimaculatus Deg. was maintained and infected with $P$. grylli as described by Sokolova et al. (2003). Briefly, the insects were infected with highly diluted water suspension of spores (approximately $10^{4}-10^{5}$ spores per $\mathrm{ml}$ ) used instead of water supply for 2nd-3rd instar nymphs during 1-2 days. On the average, it took 7-9 weeks to obtain heavily infected crickets with the fat body filled with the next generation of spores.

Purification of spores. Each infected cricket was dissected individually (Fig. 1) and its fat body was homogenized in water with a Teflon pestle in a separate Eppendorf tube. The homogenate was spun at $500 \mathrm{~g}$ for $5 \mathrm{~min}$. The white layer at the bottom of the tube, containing mature spores, was transferred to another Eppendorf tube and washed 5 times with distilled water. To ensure complete elimination of host cell debris, the spores were additionally purified by centrifugation through $70 \%$ Percoll and washed twice with distilled water.

Preparation of PFGE plugs. The protocol for preparation of plugs with the spores of $P$. grylli was described earlier (Nassonova et al. 1998, Sokolova et al. 1998). Briefly, a spore sample purified from a single cricket (ca $2-3 \times 10^{8}$ spores) was mixed with $1 \%$ SeaPlaque GTG agarose (BioWhittaker Molecular Applications, USA) cooled to $37^{\circ} \mathrm{C}$ in a plug mould. The plugs were chilled on ice and incubated in $3 \%$ $\mathrm{H}_{2} \mathrm{O}_{2}, 0.1 \mathrm{M} \mathrm{KCl}$ for $5 \mathrm{~min}$ at $0^{\circ} \mathrm{C}$ to initiate spore extrusion. Then the plugs were washed and incubated in $\mathrm{H}_{2} \mathrm{O}$ for $1 \mathrm{~h}$ at room temperature. Further cell lysis and extraction of chromosomal DNAs were performed in lysis buffer $(50 \mathrm{mM}$ TrisHCl, $100 \mathrm{mM} \mathrm{Na} 2$ EDTA, pH 9.4, 40\% DMSO, $0.5 \%$ sodium Nlauroylsarcosine) for $12 \mathrm{~h}$ at room temperature and in ESP buffer (500 mM Na 2 EDTA, pH 9.4, $1 \%$ sodium N-lauroylsarcosine, $2 \mathrm{mg} / \mathrm{ml}$ proteinase $\mathrm{K}$ ) for $48 \mathrm{~h}$ at $50^{\circ} \mathrm{C}$.

PFGE. "Contour-clamped homogeneous electric field" type of PFGE was performed using either Gene Navigator ${ }^{\mathrm{TM}}$ (Pharmacia) or CHEF-DR ${ }^{\circledR}$ III (Bio-Rad) system in $1.2 \%$ agarose gel in $0.5 \times \mathrm{TBE}$ buffer at $10^{\circ} \mathrm{C}$. Chromosomal DNAs of Saccharomyces cerevisiae YNN295 (Amersham Pharmacia Biotech) and lambda ladder PFG markers (New England BioLabs) were used as size standards. Different electrophoretic conditions were applied to optimize the fractionation of chromosomal DNAs from different zones of the karyotype.

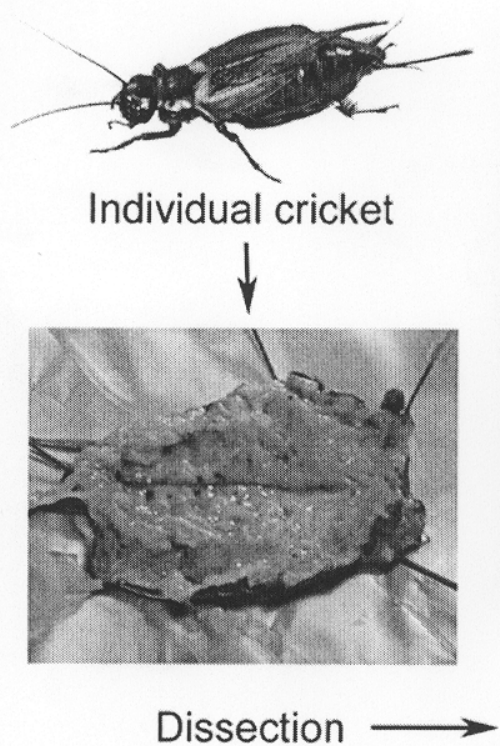

Dissection

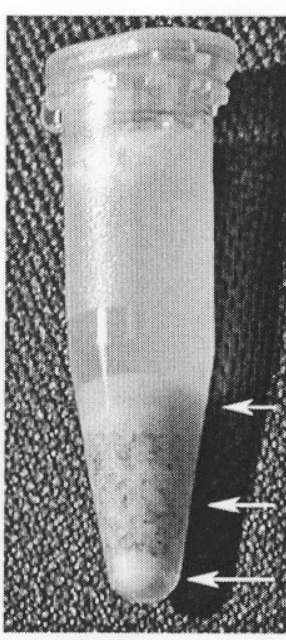

Fractionation

by

centrifugation
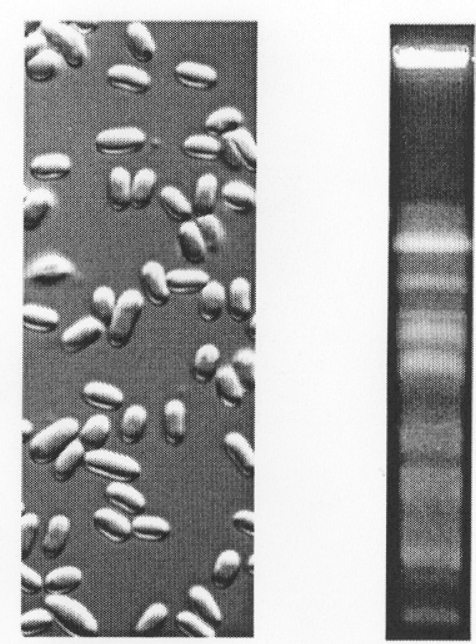

Proliferative stages

Fat body debris Spores

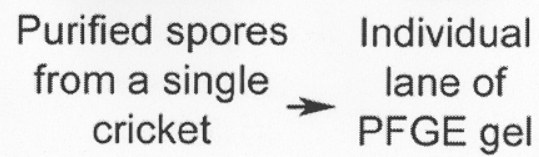

Fig. 1. Scheme illustrating the preparation of samples for PFGE and KARD 2-D PFGE analysis. The crickets were dissected individually. Spores were purified from the homogenized fat body by multi-step centrifugation. The amount of spores isolated from a single cricket $\left(2-3 \times 10^{8}\right)$ was enough to prepare a plug, so that each lane of PFGE gel represents the integral molecular karyotype of a parasite infrapopulation from one insect. 
The full set of chromosomal DNAs from the molecular karyotype of $P$. grylli was visualised in a PFGE run at $6 \mathrm{~V} / \mathrm{cm}$ either with continuous 1 to $50 \mathrm{sec}$ switch time ramp for $24 \mathrm{~h}$ (Fig. 2A), or with discrete increment of switch time: $12 \mathrm{sec}$ for 15 $\mathrm{h}$, followed by $20 \mathrm{sec}$ for $12 \mathrm{~h}$ and $40 \mathrm{sec}$ for $10 \mathrm{~h}$ (Figs. 3, $4 \mathrm{~A})$. For optimal resolution of the smallest chromosomal DNAs in the lower zone of molecular karyotype (size range from 135 to $220 \mathrm{~kb}$ ), the run time was $24 \mathrm{~h}$ at $6 \mathrm{~V} / \mathrm{cm}$ with a discrete increment ( $1 \mathrm{sec}$ every $4 \mathrm{~h}$ ) of switch time from 10 to $15 \mathrm{sec}$ (Fig. 2B). For fractionation of the molecules in the middle zone of the molecular karyotype (size range from 220 to $325 \mathrm{~kb}$ ) PFGE run was carried out for $40 \mathrm{~h}$ at $6 \mathrm{~V} / \mathrm{cm}$ with a $15 \mathrm{sec}$ switch time (Fig. 2C). Separation of the chromosomal DNAs from both the lower and the middle zones of the karyotype was performed for $42 \mathrm{~h}$ at $4.5 \mathrm{~V} / \mathrm{cm}$ with a discrete increment ( $1 \mathrm{sec}$ every $7 \mathrm{~h}$ ) of switch time from 15 to $20 \mathrm{sec}$ (Fig. 4E). To achieve the resolution in the upper zone of the molecular karyotype (size range from 325 to $485 \mathrm{~kb}$ ), the following running conditions were applied: $50 \mathrm{~h}$ at $4.5 \mathrm{~V} / \mathrm{cm}$ with 40 to $50 \mathrm{sec}$ switch time ramp (Figs. 2D, 4B). The gels were stained for $30 \mathrm{~min}$ in ethidium bromide $(0.5 \mu \mathrm{g} / \mathrm{ml})$ and recorded with a Gel Documentation and Analysis System (Herolab).

KARD 2-D PFGE. The chromosomal DNAs derived from the first-dimensional PFGE step were in-gel digested with rare-cutting endonuclease MluI (Amersham Pharmacia Biotech) and labeled with $\left[\alpha-{ }^{32} \mathrm{P}\right] \mathrm{dATP}$ according to the standard protocols (Brugère et al. 2000a, b). The resulting radiolabeled restriction fragments were PFGE-separated in the second dimension in $1.2 \%$ agarose gel in $0.5 \times$ TBE buffer at $8^{\circ} \mathrm{C}$. The run time was $24 \mathrm{~h}$ at $6 \mathrm{~V} / \mathrm{cm}$ with a $1.2 \mathrm{sec}$ switch time for 12 $\mathrm{h}$, followed by a $2.5 \mathrm{sec}$ switch time for $12 \mathrm{~h}$. For visualising radioactive DNA, the gels were vacuum-dried and placed against a Biomax ${ }^{\mathrm{TM}} \mathrm{MS}$ film (Kodak) between two intensifying screens at $-80^{\circ} \mathrm{C}$.

Hybridisation. Beta tubulin DNA probe was synthesized using a PCR procedure. A 1,158 bp fragment of beta tubulin gene of $P$. grylli was amplified using the primer pair btub1 (5'-YTGCAGACRGGRCARTGYGGRAAYC-3') and btub2 (5'-CTCNGARAACTCCATCTCRTCC-3'). Reactions were run in a Perkin Elmer Cetus thermocycler for 35 cycles, each with $94^{\circ} \mathrm{C}$ for $30 \mathrm{sec}, 50^{\circ} \mathrm{C}$ for $30 \mathrm{sec}$ and $72^{\circ} \mathrm{C}$ for $1 \mathrm{~min}$ followed with a final cycle at $72^{\circ} \mathrm{C}$ for $10 \mathrm{~min}$. PCR products were cloned in $\mathrm{pGEM}^{\circledR}$-T Easy vector (Promega). The corresponding inserts were labeled with $\left[\alpha-{ }^{32} \mathrm{P}\right] \mathrm{dCTP}$ by random priming. Radiolabeled probe was hybridized with PFGEseparated chromosomal DNAs after transfer onto Hybond-N+ nylon membranes (Amersham Pharmacia Biotech) and with restriction fragments directly in dried gels as described in Brugère et al. (2000b). Hybridisations were carried out at $65^{\circ} \mathrm{C}$ for $12 \mathrm{~h}$ in $5 \times \mathrm{SSC}, 0.5 \%$ SDS and $5 \times$ Denhardt's solution. After hybridisation, blots and gels were exposed to Biomax MS films (Kodak).

Image cytometry. The spores of $P$. grylli were air-dried on glass slides and fixed with methanol. Hydrolysis of samples was carried out at room temperature in $6 \mathrm{~N} \mathrm{HCl}$ for 6 min, then the slides were stained with $0.3 \%$ Schiff-type fluorescent dye auramine $\mathrm{O}-\mathrm{SO}_{2}$ for $1.5 \mathrm{~h}$ at $4^{\circ} \mathrm{C}$. Quantitative fluorescence image analysis was performed on a Zeiss Axioscope microscope equipped with Chiper CPT camera and interfaced with an image analysis system VideoTest (Ista, St. Petersburg). The diplokarya focused in the captured images were outlined on the screen and the values of the integrated intensity of fluorescence (IIF) were recorded. Calculation of DNA femtogram values was based on the ratio between IIFs of the diplokarya and metaphase human chromosomes used as an internal standard (chromosome I - $513 \mathrm{fg}$, chromosome II $503 \mathrm{fg}$; Mayall et al. 1984, Cram et al. 1994). Femtogram values were converted into $\mathrm{Mb}$ with the formula: genome size $(\mathrm{Mb})=0.980 \times$ DNA content $(\mathrm{fg})$ (Cavalier-Smith 1985).

\section{RESULTS}

\section{Molecular karyotype}

The molecular karyotype of $P$. grylli exhibited a complex pattern consisting of numerous bright and faint bands of variable staining intensity (Fig. 2). The chromosome sizes varied within a fairly narrow range from 135 to $485 \mathrm{~kb}$. Due to co-migration of many similarsized molecules it was difficult to resolve all chromosomal DNAs in a single separation step. Several variants of PFGE conditions were adapted to improve the resolution of particular zones of the molecular karyotype, as exemplified in Fig. 2 (B-D). Analysis of the entire set of electrophoregrams allowed us to recognize no less than 18 major and 13 minor bands. Staining intensities varied between different major bands as well as between different minor ones. Nonstoichiometric staining intensities of the bands probably reflected the presence of multiple chromosomal DNAs in some of them. Some bands probably contained more than one type of chromosomal DNAs of the same size or the greater number of copies of the same type. Therefore PFGE data did not allow for exact determination of the chromosome number in the karyotype of $P$. grylli.

Repeated PFGE analysis over a 7-year period failed to reveal any significant change in the pattern of major bands. The number of minor bands varied slightly depending on a spore sample. Karyotypes from different parasite infrapopulations (we use the term "infrapopulation" to denote a parasite population isolated from one cricket) sampled in one cage with crickets shared basically the same major band pattern, while the set of minor bands was rather variable (Fig. 3).

\section{KARD 2-D PFGE analysis of the genome}

No less than 17 variants of the pattern were distinguished in the autoradiographs of in-gel labeled and MluI digested chromosomal DNAs. These variants were assigned to 17 putative linkage groups assuming that each individual chromosome exhibited the unique pattern of restriction fragments and that the homologous chromosomes had identical or very similar ones. The putative linkage groups were designated with consecutive Roman numerals beginning with I for the smallest one. Each group comprised from one to four differentsized homologous chromosomes indicated with the indexes from "a" to "d" respectively (Fig. 4). 

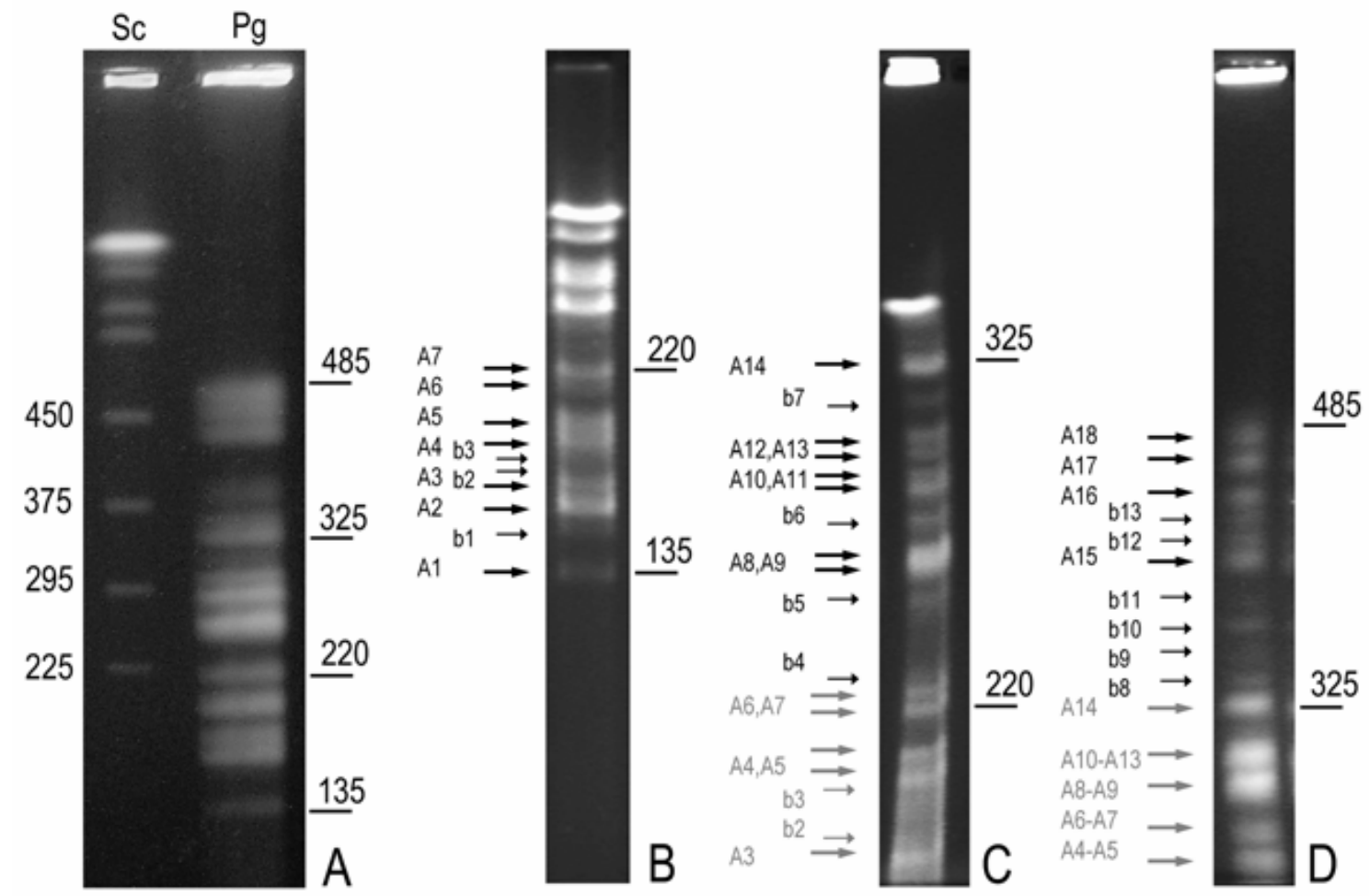

Fig. 2. Molecular karyotype of Paranosema grylli. A - PFGE separation of a full set of chromosomal DNAs of P. grylli (Pg) showing a pattern of bands sized between 135 and $485 \mathrm{~kb}$. Individual bands remain poorly resolved. The sizes of the four smallest chromosomes of Saccharomyces cerevisiae YNN 295 (Sc) used as a size standard are indicated on the left. Size markers on the right show the ranges of three conventional zones of the karyotype of $P$. grylli: lower (135-220 kb), middle (220-325 kb) and upper (325-485 kb) one. B-D - PFGE separations under conditions optimized for each of three zones. In all zones highly complex pattern of chromosomal bands of variable staining intensities was observed. Not less than 18 major (A1-A18, thick arrows) and 13 minor bands (b1-b13, thin arrows) were identified. Chromosomal bands of a given zone are labeled in black, other visible bands are indicated in grey.

KARD 2-D PFGE analysis highlighted several remarkable features of the chromosomal composition in $P$. grylli. Many adjacent bands varying in size from 5 to $40 \mathrm{~kb}$ were found to be doublets, triplets or sometimes tetrads of presumed homologues with identical or very similar restriction patterns (Table 1, Fig. 4). In most cases multiple homologues presented in presumably equimolar proportions, however sometimes relative intensity of homologous patterns varied considerably. Occurrence of size variants of homologous chromosomes presented in lesser proportions proved to explain the origin of the greater part of minor bands (e.g. bands b1, b4, b8, b9, b11, b12, b13). Molecules with different restriction patterns (i.e. heterologous molecules) often co-migrated in one major band, e.g. chromosomes Ic, IIa, IIIa in band A2; chromosomes IIb, IIIb, IVa in band A3 etc. Minor bands adjacent to such major ones represented size variants (seemingly equimolar) of one of comigrating chromosomes (e.g. bands b2, b3, b5). Therefore, KARD 2-D PFGE data demonstrated that the puzzling pattern of molecular karyotype of $P$. grylli can be explained both by the presence of multiple size variants of homologous chromosomes and by co-migration of similar-sized heterologous chromosomes.
In Southern hybridisation experiments a probe to beta-tubulin detected two discrete adjacent bands (280 and $290 \mathrm{~kb}$ ) assigned to the different-sized variants of chromosome XII. In a dried gel after KARD 2-D PFGE this probe hybridized only with two spots (Fig. 5). Betatubulin is seemingly a single-copy gene in microsporidia. Therefore, these results confirm the presence of functionally homologous chromosomes migrating in different bands.

Size variation between homologous chromosomes of $P$. grylli is related to the length polymorphism affecting only few restriction fragments. In most cases just one or two fragments exhibited polymorphism. Sometimes no differences were found in the patterns of distinct-sized homologous chromosomes. This was probably due to rather minor variations in size of polymorphic homologues, poor visualisation of low molecular weight fragments (less than $5 \mathrm{~kb}$ ) and undetected tiny differences in the size of larger fragments. Overlapping spots from the patterns of co-migrating chromosomes might also involve some difficulties in interpretation of the patterns and detection of the individual spots.

Chromosomes of the upper zone of the karyotype exhibited distinct but rather similar patterns of restriction 


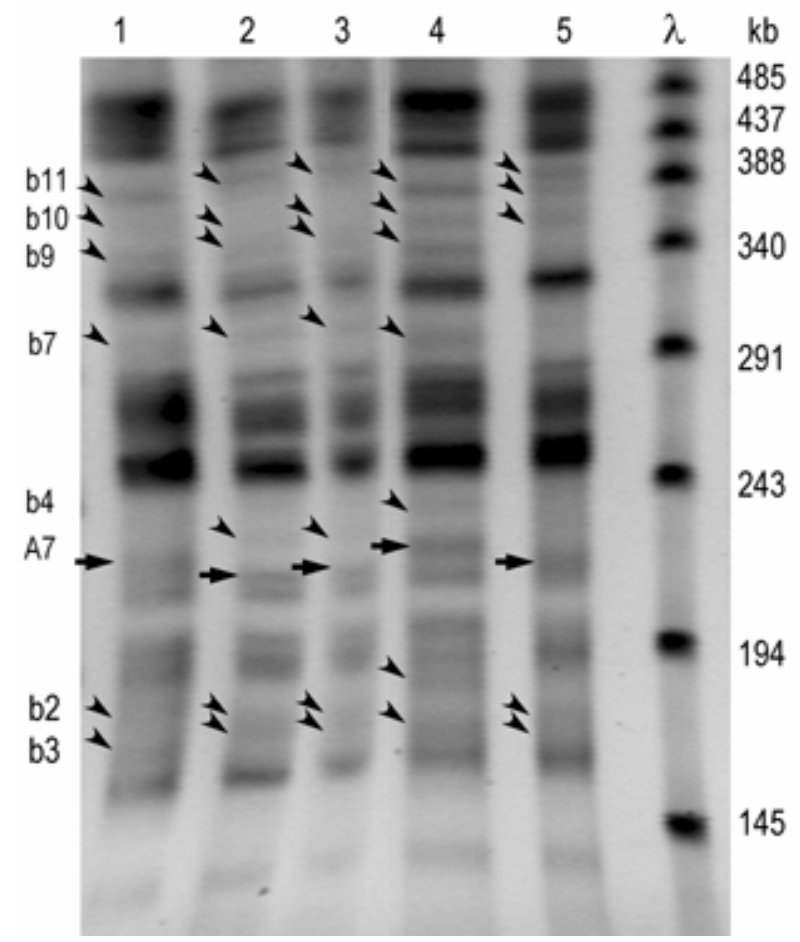

Fig. 3. Chromosomal heterogeneity among Paranosema grylli infrapopulations (1-5) isolated from the same laboratory culture. Image is inverted for better visualisation of differences in the banding patterns. Sizes of concatamers of lambda DNA $(\lambda)$ are indicated on the right. Arrowheads indicate variable minor bands (b2, b3, b4, b7, b9, b10, b11). Arrows show the only variable major band (A7). Note the following differences in the banding patterns: (1) different migration of the minor bands b2, b3, b4 and the major band A7; (2) disappearance of the minor bands $\mathrm{b} 4$ and $\mathrm{b} 7$ in some lanes, e.g. b4 is not visualised in lanes 1 and 5, b7 is missing in lane 5; (3) variability of staining intensity of the particular bands in a set of minor bands b9, b10, b11 in the upper zone of the molecular karyotype.

fragments. For example, the patterns of chromosomes $\mathrm{XIV}, \mathrm{XV}, \mathrm{XVI}$ and, to a lesser extent, those of chromosomes XIII and XVII contained from 30 to $60 \%$ fragments of the same size (Fig. 4 C, D). The similarity of patterns might result from sequence homology of lengthy regions of the chromosomes. In other words these chromosomes probably share some homologous regions.

It is noteworthy that relative intensities of 2-D spot patterns varied between different heterologous chromosomes presented by the same number of size variants. For example, chromosomes VII and VIII are the only ones that are non-polymorphic in the karyotype of $P$. grylli. However, the spots in the pattern of chromosome VII were less intensive than those in the pattern of chromosome VIII. Relative intensity of the patterns of triplets Ia-c, XIa-c, XIIIa-c and XIVa-c differed noticeably as well. The same feature was also observed in the patterns of various doublets (Fig. $4 \mathrm{C}-\mathrm{G}$ ).
Table 1. Assignment of individual chromosomes to the bands of the molecular karyotype of Paranosema grylli based on KARD 2-D PFGE data.

\begin{tabular}{|l|l|}
\hline Band* & Chromosome** \\
\hline A1 & Ia \\
b1 & Ib \\
A2 & Ic, IIa, IIIa \\
A3 & IIb, IIIb, IVa \\
b2 & IVb \\
b3 & Va \\
A4 & Vb, VIa \\
A5 & VIb, VII \\
A6 & VIII \\
A7 & IXa \\
b4 & IXb \\
b5 & Xa \\
A8 & IXc, Xb \\
A9 & IXd \\
b6 & XIa \\
A10 & XIb \\
A11 & XIIa \\
A12 & XIc \\
A13 & XIIb \\
b7 & non-identified \\
A14 & XIIIa \\
b8 & XIIIb \\
b9 & XIVa \\
b10 & XIVb \\
b11 & XIVc \\
A15 & XVa \\
b12 & XVb \\
b13 & XVc \\
A16 & XVIa \\
A17 & XVIb, XVIIa \\
A18 & XVIIb \\
\hline & \\
\hline
\end{tabular}

*Major bands are designated with uppercase letter "A" with consecutive numerical indexes beginning with A1 for the bottommost band in the lower zone of the molecular karyotype and ending with A18 for the topmost band in the upper zone. Minor bands are designated in the same way with lowercase letter "b" with consecutive numerical indexes (b1-b13). $* *$ Chromosome designation is explained in the legend to Fig. 4.

\section{Genome size and DNA content}

KARD 2-D PFGE data suggest a haploid set of 17 putative chromosomes in P. grylli. Summing the sizes of chromosomes, the haploid genome size of this microsporidium was estimated to be 4.5-4.7 Mb (Table 2). To estimate the genome ploidy in P. grylli, we performed cytometric measurements of DNA content in its spores. Two distinct zones of fluorescence corresponding to the diplokaryon were clearly visible in the spores stained with auramine $\mathrm{O}-\mathrm{SO}_{2}$ (Fig. 6). Integrated intensities of fluorescence (IIF) of the diplokarya were compared with those of the human metaphase chromosomes used as a quantitative internal standard. Based on IIF ratio the absolute DNA content in the spores of $P$. grylli was estimated at $4.9 \pm 0.8 \mathrm{fg}$ DNA/nucleus, i.e. about $4.8 \pm$ 
0.8 Mb DNA/nucleus (Table 3). Therefore, a KARD 2D PFGE estimate of the haploid genome size of P. grylli generally agrees with the image cytometry data on the absolute DNA content in the individual nuclei of diplokaryon in the spores. This suggests a haploid state of each member of the diplokaryon.

\section{DISCUSSION}

Two main factors contribute to the karyotype complexity in Paranosema grylli. These are the co-migration of heterologous molecules of the same size and the remarkable size variation of homologous chromosomes revealed by KARD 2-D PFGE and confirmed by Southern hybridisation. The most important result of the present study is the demonstration of the extensive chromosome-length polymorphism in the genome of $P$. grylli. To understand the background of this phenomenon we should consider the population structure of the parasite, its nuclear organisation, ploidy status, and reproductive strategy.

\section{Population structure}

The observed molecular karyotype resulted from superimposition of individual karyotypes from $2-3 \times 10^{8}$ spores that were isolated from one host insect. It is unknown if such infrapopulations of $P$. grylli are clonal or mixed. This microsporidium has been continuously maintained in an experimentally infected cricket culture since 1994. Initially we supposed that the numerous passages of $P$. grylli would result in selection and finally produce a clonal isolate. However, variations found in the banding patterns of the karyotypes from different infrapopulations of $P$. grylli indicated that some chromosomal heterogeneity existed in our laboratory population of this parasite.

Our data suggested that sizes and/or a number of chromosomes migrating in the minor bands varied among infrapopulations. The major bands remained invariable (Fig. 3). KARD 2-D PFGE analysis demon- strated that the minor bands consisted of chromosomal DNAs originating from a single chromosome, while the major bands were the mixture of DNAs originating from homo- and heterologous chromosomes of similar size. Size difference among DNAs migrating in a major band was $5-20 \mathrm{~kb}$. The size variation among the polymorphic homologues was within the same range. Thus the variability of chromosomes in the major bands probably remained hidden when infrapopulations were compared by PFGE.

It is unclear if an infrapopulation itself comprises several descendant subpopulations with different-sized variants of the same chromosome. At present investigations relevant to this question are seriously hampered by the absence of techniques of clone selection developed for $P$. grylli. Infestation of the adipose tissue at advanced stages of propagation of $P$. grylli in a cricket occurs without any apparent clustering of the parasites, which does not allow for isolation of separate parasite subpopulations of a presumed clonal origin. Further exploration of chromosomal heterogeneity in P. grylli requires finding an approach to evade this problem.

\section{The ploidy status of the diplokaryon}

There are two opposing views on the ploidy of the diplokaryon: diploidy versus haploidy of the individual nuclei. A diploid state of each nucleus of the diplokaryon was suggested after identification of synaptonemal complexes in meiotic diplokarya of Thelohania spp., Polydispyrenia simulii and Janacekia debaisieuxi (Loubès and Maurand 1971, Loubès 1979, Vivarès and Sprague 1979). Later, Hazard and Brookbank (1984) after microphotometric measurements of the DNA content during meiosis in Amblyospora sp. found evidences that the developmental stage harbouring synaptonemal complexes in each member of the diplokaryon could be only a brief transitional stage between the end of karyogamy and the onset of meiosis. It was postulated that the paired nuclei were haploid, except for this brief

Fig. 4. KARD 2-D PFGE analysis of the genome of Paranosema grylli. A - Molecular karyotype showing a full set of chromosomal DNAs (inverted image). Major bands are numbered on the top. B-D - KARD 2-D PFGE analysis of chromosomal DNAs in the size range from 325 to $485 \mathrm{~kb}$. E-G - KARD 2-D PFGE analysis of chromosomal DNAs in the size range from 135 to 325 $\mathrm{kb} . \mathbf{B}, \mathbf{E}$ - inverted images of the molecular karyotype derived from the first-dimensional PFGE step; $\mathbf{C}, \mathbf{F}-$ autoradiographs of radiolabeled restriction fragments separated in the course of the second-dimensional PFGE step; $\mathbf{D}, \mathbf{G}$ - interpretative diagrams of 2-D spot pattern. Each ellipse in the diagrams D and G corresponds to a detected spot containing one or more restriction fragments. Double ellipses correspond to bifocal and closely spaced spots. Array of ellipses corresponding to an ensemble of spots identified in the restriction pattern of each individual chromosome is painted with specific colour. Individual chromosomes are also indicated with respectively coloured arrows. Multiple arrows of the same colour specify the polymorphic homologues with identical or very similar patterns of restriction fragments (thin arrows show the size variants with less intense spot patterns). Deduced assignment of the linkage groups to the series of restriction fragments is indicated. Presumable linkage groups are designated with consecutive Roman numerals. Polymorphic homologues are specified with lower-case indexes. Positions of the size standards are indicated on the right of the autoradiographs $\mathrm{C}$ and $\mathrm{F}$ and of the corresponding diagrams D and G. Sizes of the chromosomal bands in the molecular karyotypes are indicated on the top of panels B and E. Dotted lines connect the corresponding chromosomal bands in panels A, B, E. Black arrow on the top indicates the direction of migration in the first-dimensional PFGE gels (A, B, E). Black arrow on the left side of panel $\mathrm{C}$ denotes the direction of migration in the KARD 2-D PFGE gels (C, F). Note the following groups of polymorphic chromosomes: doublets of homologues present in apparently equimolar proportions (IIa-b, IIIa-b, IVa-b, Va-b, VIa-b, Xa-b, XIIa-b, XVIa-b, XVIIa-b), non-equimolar doublet XIIIa-b, non-equimolar triplets Ia-c, XIa-c, XIVa-c, XVa-c and tetrad IXa-d (size variants of chromosome IX present apparently in equimolar proportions with the exception of IXb). 


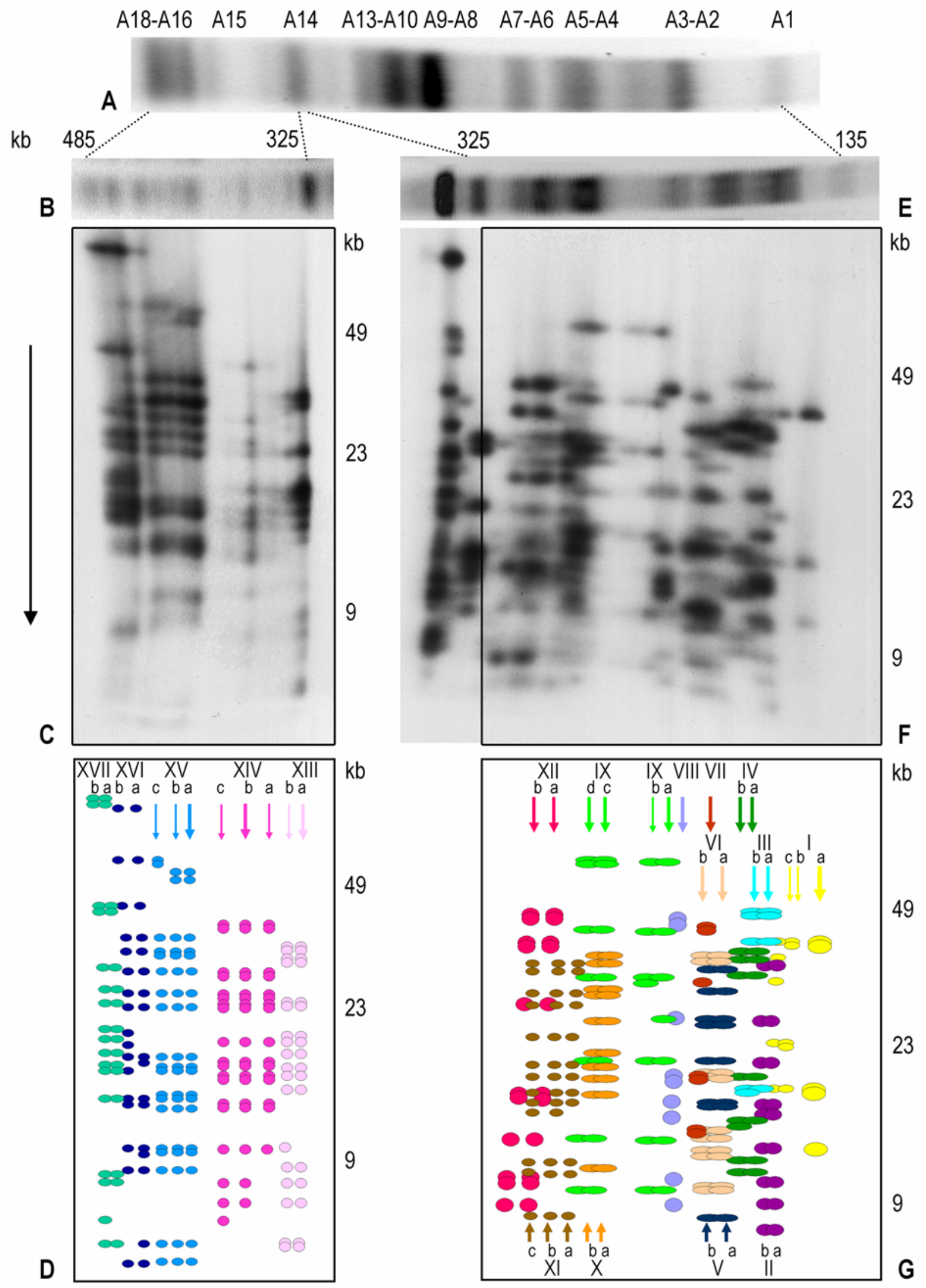




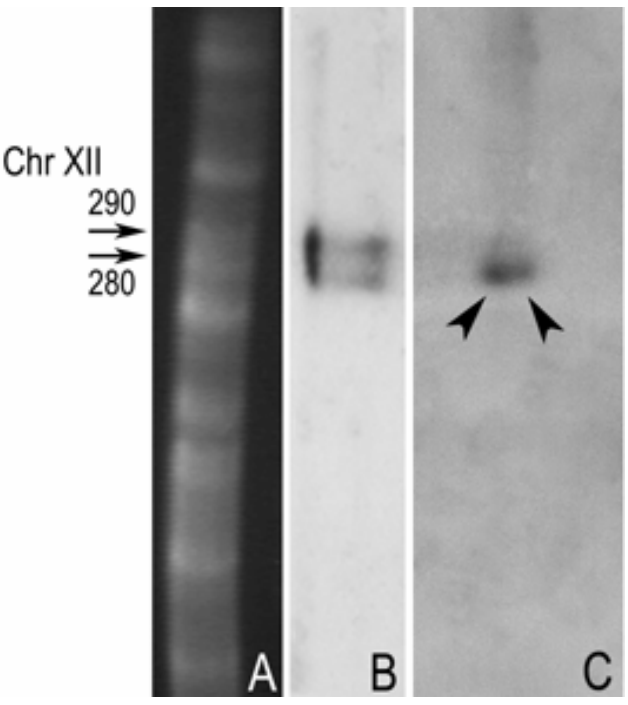

Fig. 5. Chromosomal localisation of beta tubulin gene in the genome of Paranosema grylli. A - ethidium bromide-stained gel with PFGE-separated chromosomal DNAs; B - autoradiograph of Southern blot; C - KARD 2-D PFGE dried gel hybridized with the radiolabeled beta tubulin probe. Arrows indicate hybridisation signal in Southern blot associated with two adjacent bands (280 and $290 \mathrm{~kb}$ ) assigned to differentsized variants of chromosome XII. Chromosome size and band designation are specified on the left. Arrowheads indicate the only two adjacent spots in KARD 2-D PFGE gel hybridized with beta tubulin probe.

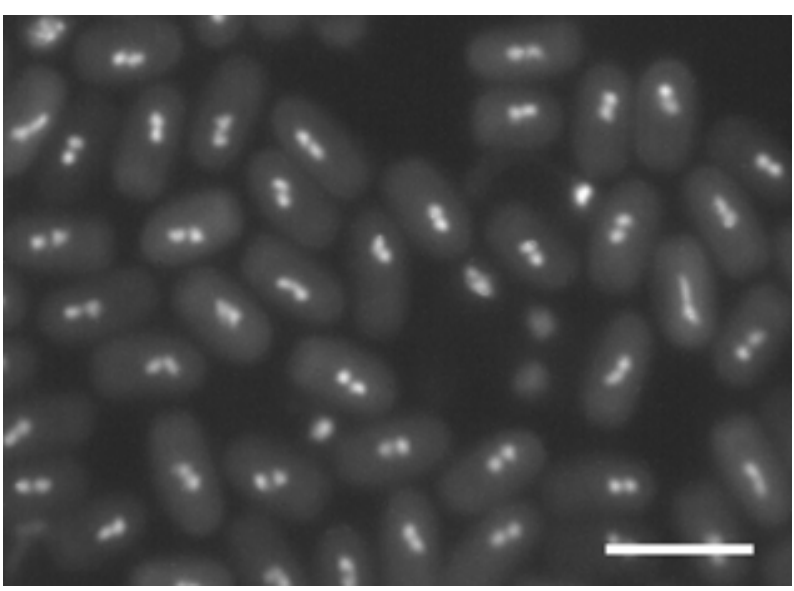

Fig. 6. Visualisation of diplokaryon in the spores of Paranosema grylli by staining with auramine $\mathrm{O}-\mathrm{SO}_{2}$. Scale bar $=5$ $\mu \mathrm{m}$.

stage. Despite many uncertainties in the scheme of meiosis proposed by Hazard and Brookbank (1984), their conception of the ploidy cycle and scheme of reductional division in microsporidia remains the most widely accepted (Canning 1988, Sprague et al. 1992).

In KARD 2-D PFGE autoradiographs of $P$. grylli we revealed multiple size variants of homologous chromosomes. If we suppose that each of the adjacent nuclei of the diplokaryon is diploid, then every chromosome is represented with four homologues. In this case the fol- lowing ratios of homologous chromosomes can be theoretically reflected in KARD 2-D PFGE autoradiographs:

(a) All the four homologues are of the same size. Thus in the autoradiograph we must see a single 2-D spot pattern.

(b) All the four homologues are of different size. They must produce an equimolar tetrad.

(c) The four homologues are distributed in three groups according to their sizes. They form a non-equimolar triplet, in which one of the three polymorphic variants is present in a double number of copies.

(d) The four homologues are distributed in two groups. Each group is present in equimolar proportions, thus producing an equimolar doublet.

(e) The four homologues are distributed in two groups, but one of these groups are present in a triple number of copies. In this case a non-equimolar doublet must appear.

In the KARD 2-D PFGE autoradiographs of P. grylli (Fig. 4) we revealed only one variant of restriction pattern for non-polymorphic chromosomes VII and VIII (case "a") and numerous doublets of homologues presenting in apparently equimolar proportions (case "d": IIa-b, IIIa-b, IVa-b, Va-b, VIa-b, Xa-b, XIIa-b, XVIa-b, XVIIa-b). The density of spots in the patterns of the doublet XIIIa-b apparently did not correspond to that expected for a "true" non-equimolar doublet (case "e"). The spots in the pattern of chromosome XIIIa is much denser than those in the pattern of XIIIb. Moreover, we did not find any equimolar tetrads (case "b"). In contrary, the only revealed tetrad is non-equimolar, with the homologue IXb presenting in obviously lesser proportions than IXa, IXc and IXd. Among the nonequimolar triplets (Ia-c, XIa-c, XIVa-c, XVa-c) at least one (Ia-c) shows gradual increment of density from one restriction pattern to another instead of showing two patterns of a similar density and one twice as dense, as it should be in the case "c". Thus, the distribution of size variants for several chromosomes is seemingly inconsistent with the hypothesis of diploidy.

\section{Chromosomal rearrangements during the parasite proliferation}

If each nucleus of the diplokaryon is haploid, there should be one or two size variants of each putative chromosome. Within this hypothesis, the greater number of size variants and the non-equimolar doublets may be attributed to recombination events and chromosomal rearrangements.

The size difference between the polymorphic homologues in P. grylli is rather small. The largest variation was observed for chromosomes IX and XIV (17\% and $10 \%$ of the mean size, respectively). For the rest of the chromosomes the size variations did not exceed $6 \%$. The patterns of restriction fragments of the size-variable homologues were identical or very similar. This observation provides evidence that the chromosomal core is 
Table 2. Sizes of chromosomes and genome size in Paranosema grylli.

\begin{tabular}{|l|c|}
\hline Chromosome & Size $(\mathrm{kb})$ \\
\hline Ia-c & $135-155$ \\
IIa-b & $160-165$ \\
IIIa-b & $160-180$ \\
IVa-b & $175-190$ \\
Va-b & $190-195$ \\
VIa-b & $195-200$ \\
VII & 200 \\
VIII & 215 \\
IXa-d & $220-260$ \\
Xa-b & $250-260$ \\
XIa-c & $265-285$ \\
XIIa-b & $280-290$ \\
XIIIa-b & $325-345$ \\
XIVa-c & $360-395$ \\
XVa-c & $420-445$ \\
XVIa-b & $455-470$ \\
XVIIa-b & $480-485$ \\
Haploid genome size* & $4,485-4,735$ \\
\hline
\end{tabular}

*Range for the haploid genome size was deduced by summing the sizes of individual chromosomes.

Table 3. DNA content in the spores of Paranosema grylli measured by image cytometry.

\begin{tabular}{|l|c|c|}
\hline \multicolumn{1}{|c|}{ Internal standard } & Ratio IIFd/IIFs* & $\begin{array}{c}\text { DNA content } \\
\text { (fg/nucleus) }\end{array}$ \\
\hline $\begin{array}{l}\text { Human metaphase } \\
\text { chromosome I }(513 \mathrm{fg})\end{array}$ & $0.0187 \pm 0.0053$ & $4.79 \pm 1.22$ \\
$\begin{array}{l}\text { Human metaphase } \\
\text { chromosome II }(503 \mathrm{fg})\end{array}$ & $0.0201 \pm 0.0045$ & $5.06 \pm 1.12$ \\
\hline \multicolumn{2}{|l|}{ Mean } & $4.94 \pm 0.82$ \\
\hline
\end{tabular}

*Ratio between integrated intensity of fluorescence of the diplokarya (IIFd) and that of the standards (IIFs). All values are given with $95 \%$ confidence interval calculated for the aggregate samples.

stable and only the extremities are affected by rearrangements. This suggests a role of ectopic recombination events in chromosomal size variation of $P$. grylli.

To date it is generally assumed that repeated sequences located at the chromosome extremities mediate ectopic recombination events between homo- and heterologous chromosomes. In parasites this mechanism allows expansion and diversification of the gene families located at the chromosome ends and involved in such mechanisms as antigenic variation, cytoadhesion, and drug resistance (Lanzer et al. 1995, Barry et al. 2003). Ectopic recombinations resulting in chromosome-length polymorphism were described in the genomes of various parasitic protists with different ploidy status, e.g. in polyploid Giardia lamblia (Le Blancq and Adam 1998), in diploid organisms (e.g. Encephalitozoon cuniculi - Brugère et al. 2001, Faye et al. 2003) and in haploid ones (e.g. Plasmodium falciparum Freitas-Junior et al. 2000).
The size variation between two homologues is generally the result of a single recombination event, namely insertion or deletion of a few $\mathrm{kb}$. In the hypothesis of haploidy of each nucleus of the diplokaryon, we can propose a model which regards the complex karyotype of $P$. grylli revealed by KARD 2-D PFGE analysis as a result of subsequent recombination events occurring during the parasite propagation in the cricket.

In KARD 2-D PFGE we analyse the cumulative karyotype of parasite infrapopulation. After infection with the microsporidium the fat body of the cricket will be progressively colonised by successive infection events. During the proliferation a recombination event may occur in one parasite. It will start a subpopulation of parasites bearing a new size variant of affected chromosome. Thus the entire infrapopulation of $P$. grylli in a given cricket will become heterogeneous. The earlier the rearrangement occurs during the parasite propagation, the higher the proportion of recombinant subpopulation will be, and therefore the denser the spot pattern corresponding to this recombinant chromosome will occur in KARD 2-D PFGE autoradiograph.

The DNA bands in PFGE gel became visible if $10^{8}$ or more spores were used to prepare a plug. Thus, only the DNA molecules numerous enough to produce a visible band can be detected. The amount of spores isolated from a single cricket is $2-3 \times 10^{8}$. Even if no more than $30 \%$ of the initial amount of spores remained after purification, we can assume that the spore production in our experiments did not exceed $10^{9}$ spores/cricket. The number of generations required to reach this value can be calculated as $\log _{2} 10^{9}$, and equals to 30 generations, while the number of generations required to reach the detection limit of $10^{8}$ is about 27. Thus, to reach the number of copies exceeding the $10^{8}$ barrier, all chromosomal variants that we were able to detect in KARD 2-D PFGE must originate during the several initial generations of the parasite. This increases the chances that they are derived from one or few parasites that first started proliferation in a cricket. Even if recurrent infestations in the cricket population happened later, their influence would remain undetectable. To infect the cricket we used a suspension containing ca $10^{4}-10^{5}$ spores per $\mathrm{ml}$. From our experience, this is the minimal amount required to infect a cricket. This further increases the chances that the observed chromosome composition is derived from a single ancestor. We proceed from this assumption to simplify the suggested model.

In the absence of recombination we should reveal in KARD 2-D PFGE autoradiograph only one variant of spot pattern if the chromosome is non-polymorphic, and an equimolar doublet if it is represented by two sizevariants originating from the different nuclei of the diplokaryon. In the case of a recombination event we would observe a non-equimolar doublet or a triplet of homologues. If two recombination events subsequently 
involve the same chromosome, a non-equimolar triplet or a tetrad of homologues will be produced.

The estimated number of recombination events for the infrapopulation analysed in Fig. 4 can be as follows:

(a) No recombination for the non-polymorphic chromosomes VII and VIII and for equimolar doublets of chromosomes IIa-b, IIIa- $-\mathrm{d}$, IV $\mathrm{a}-\mathrm{b}, \mathrm{Va}-\mathrm{b}$, VIa-b, Xa-b, XIIa-b, XVIa-b and XVIIa-b (presumably a result of recombinations that took place during the previous developmental cycles of the parasite, before it infected the cricket investigated).

(b) One recombination for chromosomes XIa-c (a recent event resulting in the lower density of the spot pattern of XIa as compared with those of XIb and XIc, which are nearly equal), XIIIa-b (probably a very recent event, since the density of spots in the pattern of chromosome XIIIa is much higher than that in the pattern of XIIIb), XIVa-c and XVa-c (the recombination events at the early beginning of infection can explain the observed triple pattern, in which one of the three 2-D spot patterns is approximately twice as intense);

(c) Two recombinations for chromosomes Ia-c (triplet with gradual increment of density from one restriction pattern to another: $\mathrm{Ia}>>\mathrm{Ib}>\mathrm{Ic}$ ) and $\mathrm{IXa}-\mathrm{d}$ (pattern of IXb is less intense than the patterns of IXa, IXc, IXd; this non-equimolar tetrad may result from one distant event at the early beginning of parasite proliferation and one more recent event).

In all, eight recombination events should be assumed for interpretation of the chromosomal composition observed in Fig. 4. A similar model can be compiled for the case of diploid nuclei of the diplokaryon, but in this case we would have more than four homologues as frequent as the other combinations described above. We have never observed more than four size-distinct homologues in any of samples analysed by KARD 2-D PFGE (data not shown). Therefore, within the proposed model KARD 2-D PFGE data suggest a haploid state of the individual nuclei of the diplokaryon in P. grylli.

The KARD 2-D PFGE estimate of the haploid genome size of $P$. grylli is in agreement with the image cytometry data of the absolute DNA content in the individual nuclei of the diplokaryon. The estimates of DNA content in spores of $P$. grylli have to be accepted as "ball-park" figures due to relatively low accuracy of image cytometry measurements in such a minute object as a microsporidian cell. The accuracy is hampered by several factors including non-specific background fluorescence commonly observed in microbial cells, photobleaching effect, limits of resolution of the method (ca $2.4 \mathrm{fg}$, according to Mayall et al. 1984), and variation among estimates provided by different authors for the common DNA quantitative standards. Nevertheless, consistency of KARD 2-D PFGE analysis and image cytometry data should be taken into account and can be considered as one more argument in favour of the haploidy hypothesis.

The proposed model can explain many puzzling features of the chromosomal composition in P. grylli. However, the unequal intensity of spot patterns of heterologous chromosomes occurring in the same number of size variants (even in case of the non-polymorphic chromosomes VII and VIII) remains mysterious. A reliable method for precise measurement of the spot density in KARD 2-D PFGE autoradiographs should be developed. This would facilitate estimation of the relative proportion of each chromosome as well as of each size variant for polymorphic homologues, and therefore would promote verification of the proposed model and further studies of the ploidy status and frequency of recombination of the individual chromosomes in the karyotype of $P$. grylli.

\section{Reproductive strategy of Paranosema grylli}

The life cycle of monomorphic diplokaryotic microsporidia involves two major phases: proliferation, during which diplokaryotic meronts and sporonts multiply (usually by binary fission), and sporogenesis, leading to the formation of diplokaryotic spores. There is no evidence for a sexual process and ploidy reduction in this cycle. It is considered to be an asexual remnant of a more complex ancestral life cycle with meiosis and alternation of ploidy phases, characteristic of polymorphic species (Sprague 1978, Loubès 1979, Issi 1981, Baker et al. 1997, Sokolova and Issi 1997).

The diplokaryon divides equationally, so that each nucleus of a pair contributes to only one daughter nucleus of the daughter diplokaryon (Vávra 1976, Toguebaye and Marchand 1984). In the case of non-random nuclear distribution and in the absence of karyogamy, mutations should accumulate independently in each nucleus. According to Cavalier-Smith (1995), "a diplokaryotic cell will evolve to comprise genetically divergent nuclei, which would be in effect two different clonal lineages within the same cell". Given that internuclear exchange is absent or rare in monomorphic diplokaryotic microsporidia, it could be possible to suggest that the chromosome organisation may be strongly altered, and therefore the homologous chromosomes originating from the different nuclei of the diplokaryon may show structural divergence.

One of the most remarkable features of the chromosomal composition of $P$. grylli is the high proportion of equimolar doublets of homologues. We suggested that they corresponded to the polymorphic homologues originating from the different nuclei of the diplokaryon. Internuclear chromosome-length polymorphism was described, for example, in dikaryotic fungi during vegetative growth and in the diplomonad Giardia lamblia. When two nuclei of the dikaryon of the white rot fungus Pleurotus ostreatus were separated by protoplasting, the comparative analysis of karyotypes from the proto- 
clones (monokaryotic mycelia with nuclear constitution identical to either one of the parental nuclei of the dikaryon) revealed length polymorphism of almost all homologous chromosomes (Larraya et al. 1999). In the asexual binucleate G. lamblia, chromosome size heterogeneity is well documented. However, allelic heterozygosity at the sequence level in this organism appeared to be very low, suggesting the existence of some mechanisms to maintain homozygosity between the two nuclei (Le Blancq and Adam 1998, Adam 2000).

The conserved restriction patterns of homologous chromosomes revealed by KARD 2-D PFGE analysis in $P$. grylli suggest that discrete events like short insertions or deletions, which do not affect the chromosome core, are responsible for chromosome-length polymorphism in this microsporidium. This observation suggests that despite the length polymorphism the sequence divergence between homologous chromosomes cannot be high. Progressive chromosome alternation and divergence between the nuclei should have been observed in the molecular karyotype as a Gaussian distribution of the positions for each chromosome. We would have had no clear band pattern in this case. However, the molecular karyotype of $P$. grylli consists of discrete bands. Therefore, our observations are in contradiction with the expected divergence of different nuclei of the diplokaryon. It suggests that some mechanisms homogenizing the genome between the nuclei of the diplokaryon in P. grylli do exist.

Since its original description (Sokolova et al. 1994), P. grylli has been considered diplokaryotic throughout its entire life cycle, and therefore asexual. Redescription of this species revealed some deviations from the typical developmental sequence of monomorphic diplokaryotic microsporidia, suggesting the existence of a brief unikaryotic stage at the end of merogony, but no evidence of meiosis has been reported (Sokolova et al. 2003). Only recently, synaptonemal complexes and a diplokaryon configuration characteristic of the onset of meiosis were observed in thin sections of the early proliferative stages (Nassonova and Smirnov 2005). This indicates the occurrence of a process similar to the meiosis reported in polymorphic and some monomorphic monokaryotic microsporidia. However, the position of meiosis in the life cycle of $P$. grylli, its products, and the following post-meiotic steps remain unclear. It should be stressed that meiosis-related structures were identified in $P$. grylli for the first time after ten years of regular investigations of this species continuously maintained in the laboratory culture. There are two alternate explanations why meiosis in $P$. grylli failed to be detected until now: (1) meiosis occurs on one of the early phases of development and propagation in the host, which are still poorly known, or (2) it is a facultative part of the life cycle. Asexual reproduction with infrequent rounds of sexual recombination is characteristic of many protists. Several unicellular organisms, once considered to be entirely asexual, were after more careful examination found to undergo meiosis, which occurred too infrequently to be easily detected (Dacks and Roger 1999).

Extensive karyotypic variability is believed to be a reflection of complete lack or rarity of sexual process, since polymorphic homologues might fail to pair during meiosis (Kistler and Miao 1992, Fierro and Martin 1999). We assume that chromosome-length polymorphism in P. grylli does not affect the core of the chromosomes and thus does not hamper the chromosome pairing in meiosis. Therefore it probably does not inhibit the meiosis-associated process of genome homogenisation. The frequency and consequences of meiosis in the life cycle of $P$. grylli need to be clarified to evaluate its role in genome shaping.

The extensive chromosome-length polymorphism described in $P$. grylli indicates the genome plasticity in this microsporidium. Genome plasticity is thought to be a dynamic response to the environmental pressure, which leads to the evolutionary adaptation (Iovannisci and Beverley 1989, Ravetch 1989, Janbon et al. 1998). We suppose that a similar phenomenon may be found in other monomorphic diplokaryotic species. One of the primary goals in further exploration of chromosomelength polymorphism and genome dynamics in P. grylli is the study of chromosome extremities and analysis of distribution of repeated sequences in the genome of this organism.

Acknowledgements. This study was supported by a program of the French Ministry for Higher Education and Research and by RFBR (Russian Foundation for Basic Research) grants no. 04-04-49209 and 05-04-49222. E.N. was supported by personal fellowship (M00-2.6K-682) for young scientists from Committee on Science and Higher Education, Administration of St. Petersburg. We are very grateful to two anonymous reviewers for valuable comments and suggestions. We would like to thank Yuri Tokarev for the help with cricket culture maintenance and Brigitte Chebance, Roland Guerry and Ivan Wrawziniak for technical assistance. We are indebted to Irma Issi and Alexey Smirnov for critical reading of the manuscript and to Oleg Manylov for language corrections. We are grateful to Julia Sokolova and Slava Dolgikh for encouragements and helpful discussions. E.N. wants to thank Lamjed Mansour for advice and technical assistance at the early phase of KARD 2-D PFGE analysis and Gregory I. Stein for assistance with image cytometry software. 


\section{REFERENCES}

ADAM R.D. 2000: The Giardia lamblia genome. Int. J. Parasitol. 30: 475-484.

BAKER M.D., VOSSBRINCK C.R., BECNEL J.J., MADDOX J.V. 1997: Phylogenetic position of Amblyospora Hazard \& Oldacre (Microspora: Amblyosporidae) based on small subunit rRNA data and its implication for the evolution of the microsporidia. J. Eukaryot. Microbiol. 44: $220-225$.

BARRY J.D., GINGER M.L., BURTON P., McCULLOCH R. 2003: Why are parasite contingency genes often associated with telomeres? Int. J. Parasitol. 33: 29-45.

BRUGÈRE J.F., CORNILLOT E., BOURBON T., MÉTÉNIER G., VIVARÈS C.P. 2001: Inter-strain variability of insertion/deletion events in the Encephalitozoon cuniculi genome: a comparative KARD-PFGE analysis. J. Eukaryot. Microbiol. 48 (Suppl.): 50S-55S.

BRUGÈRE J.F., CORNILLOT E., MÉTÉNIER G., VIVARÈS C.P. 2000a: In-gel DNA radiolabelling and two-dimensional pulsed field gel electrophoresis procedures suitable for fingerprinting and mapping small eukaryotic genomes. Nucleic Acids Res. 28: E48.

BRUGÈRE J.F., CORNILLOT E., MÉTÉNIER G., VIVARÈS C.P. 2000b: Occurrence of subtelomeric rearrangements in the genome of the microsporidian parasite Encephalitozoon cuniculi, as revealed by a new fingerprinting procedure based on two-dimensional pulsed field gel electrophoresis. Electrophoresis 21: 2576-2581.

CANNING E.U. 1988: Nuclear division and chromosomal cycle in microsporidia. BioSystems 21: 333-340.

CAVALIER-SMITH T. 1985: Cell volume and evolution of eukaryotic genome size. In: T. Cavalier-Smith (Ed.), The Evolution of Genome Size. John Wiley and Sons, Chichester, pp. 105-184.

CAVALIER-SMITH T. 1995: Cell cycles, diplokaryosis and the archezoan origin of sex. Arch. Protistenkd. 145: 189207.

CRAM L.S., FAWCETT J., CARTER N.P. 1994: Chromosome analysis and sorting with commercial flow cytometers. In: XVII Meeting of the International Society for Analytical Cytology. Life Sciences Division and The National Flow Cytometry Resource, Tutorial IV, Append. 5.

DACKS J., ROGER A.J. 1999: The first sexual lineage and the relevance of facultative sex. J. Mol. Evol. 48: 779783.

FAYE N., CORNILLOT E., TOGUEBAYE B.S., VIVARÈS C.P. 2003: On the organization of subterminal chromosomal regions in Encephalitozoon cuniculi. J. Eukaryot. Microbiol. 50: 29A

FIERRO F., MARTIN J.F. 1999: Molecular mechanisms of chromosomal rearrangement in fungi. Crit. Rev. Microbiol. 25: 1-17.

FREITAS-JUNIOR L.H., BOTTIUS E., PIRRIT L.A., DEITSCH K.W., SCHEIDIG C., GUINET F., NEHRBASS U., WELLEMS T.E., SCHERF A. 2000: Frequent ectopic recombination of virulence factor genes in telomeric chromosome clusters of $P$. falciparum. Nature 407: 10181022.
HAZARD E.I., BROOKBANK J.W. 1984: Karyogamy and meiosis in an Amblyospora sp. (Microspora) in the mosquito Culex salinarius. J. Invertebr. Pathol. 44: 3-11.

IOVANNISCI D.M., BEVERLEY S.M. 1989: Structural alterations of chromosome 2 in Leishmania major as evidence for diploidy, including spontaneous amplification of the mini-exon array. Mol. Biochem. Parasitol. 34: 177188.

ISSI I.V. 1981: [Current state of the research on the life cycles of Microsporidia.] In: M.V. Krylov (Ed.), Evolution and phylogeny of the unicellular animals. Proc. Zool. Institute Acad. Sci. USSR, Vol. 107, Leningrad, pp. 58-73. (In Russian.)

JANBON G., SHERMAN F., RUSTCHENKO E. 1998: Monosomy of a specific chromosome determines Lsorbose utilization: a novel regulatory mechanism in Candida albicans. Proc. Natl. Acad. Sci. USA 95: 5150-5155.

KAWAKAMI Y., INOUE T., ITO K., KITAMIZU K., HANAWA C., SUNAIRI M., ANDO T., IWANO H., ISHIHARA R. 1994: Comparison of chromosomal DNA from four microsporidia pathogenic to the silkworm, Bombyx mori. Appl. Entomol. Zool. 29: 120-123.

KISTLER H.C., MIAO V.P.W. 1992: New modes of genetic change in filamentous fungi. Annu. Rev. Phytopathol. 30: 131-152.

LANZER M., FISCHER K., Le BLANCQ S.M. 1995: Parasitism and chromosome dynamics in protozoan parasites: is there a connection? Mol. Biochem. Parasitol. 70: 1-8.

LARRAYA L.M., PÉREZ G., PEÑAS M.M., BAARS J.J.P., MIKOSCH T.S., PISABARRO A.G., RAMÍREZ L. 1999: Molecular karyotype of the white rot fungus Pleurotus ostreatus. Appl. Environ. Microbiol. 65: 3413-3417.

Le BLANCQ S.M., ADAM R.D. 1998: Structural basis of karyotype heterogeneity in Giardia lamblia. Mol. Biochem. Parasitol. 97: 199-208.

LOUBÈS C. 1979: Recherches sur la méiose chez les microsporidies: conséquences sur les cycles biologiques. J. Protozool. 26: 200-208.

LOUBÈS C., MAURAND J. 1971: Remarques sur le "diplocaryon" des Microsporidies. Acad. d'Agric. de France. Extrait du process-verbal de la Séance du 8 Déc. 1971: $1540-1543$.

MALONE L., McIVOR C. 1993: Pulsed-field gel electrophoresis of DNA from four microsporidian isolates. J. Invertebr. Pathol. 61: 203-205.

MAYALL B.M., CARRANO A.V., MOORE II D.H., ASHWORTH L.K., BENNETT D.E., MENDELSOHN M.L. 1984: The DNA-based human karyotype. Cytometry 5: 376-385.

MÉTÉNIER G., VIVARÈS C.P. 2001: Molecular characteristics and physiology of microsporidia. Microbes Infect. 3: 407-415.

MUNDERLOH U., KURTTI T., ROSS S. 1990: Electrophoretic characterization of chromosomal DNA from two microsporidia. J. Invertebr. Pathol. 56: 243-248.

NASONOVA E.S., SOKOLOVA Yu.Ya., SKARLATO S.O. 1998: Chromosomal DNA and the size of the genome of 
the microsporidian Nosema grylli. Dokl. Biol. Sci. 361: 394-396.

NASSONOVA E.S., SMIRNOV A.V. 2005: Synaptonemal complexes as evidence for meiosis in the life cycle of the monomorphic diplokaryotic microsporidium Paranosema grylli. Eur. J. Protistol. 41. (In press.)

RAVETCH J.V. 1989: Chromosomal polymorphism and gene expression in Plasmodium falciparum. Exp. Parasitol. 68: 121-125.

SOKOLOVA Y.Y., DOLGIKH V.V., MORZHINA E.V., NASSONOVA E.S., ISSI I.V., TERRY R.S., IRONSIDE J.E., SMITH J.E., VOSSBRINCK C.R. 2003: Establishment of the new genus Paranosema based on the ultrastructure and molecular phylogeny of the type species Paranosema grylli Gen. Nov., Comb. Nov. (Sokolova, Selezniov, Dolgikh, Issi 1994), from the cricket Gryllus bimaculatus Deg. J. Invertebr. Pathol. 84: 159-172.

SOKOLOVA Yu.Ya., NASONOVA E.S., SOMOVA N.V., SKARLATO S.O. 1998: Ultrastructure of the nuclear apparatus and electrophoretic karyotype of the microsporidian Nosema grylli (Archezoa, Microspora) - an intracellular parasite of the cricket Gryllus bimaculatus. Tsitologiya 40: 407-416, plates III-IV. (In Russian.)

SOKOLOVA Yu.Ya., ISSI I.V. 1997: On the genus Nosema (Microsporidia) in relation to new data on a life cycle of the microsporidia N. mesnili. Parazitologiya 31: 307-313. (In Russian.)
SOKOLOVA Yu.Ya., SELEZNIOV K.V., DOLGIKH V.V., ISSI I.V. 1994: Microsporidia Nosema grylli n.sp. from the cricket Gryllus bimaculatus. Parazitologiya 28: 488494. (In Russian.)

SPRAGUE V. 1978: Characterization and composition of the genus Nosema. In: W.M. Brooks (Ed.), Selected topics on the genus Nosema (Microsporida). Misc. Publ. Entomol. Soc. Am., Vol. 11, Washington, D.C., pp. 5-16.

SPRAGUE V., BECNEL J.J., HAZARD E.I. 1992: Taxonomy of phylum Microspora. Crit. Rev. Microbiol. 18: 285-395.

STREETT D. 1994: Analysis of Nosema locustae (Microsporida: Nosematidae) chromosomal DNA with pulsedfield gel electrophoresis. J. Invertebr. Pathol. 63: 301-303.

TOGUEBAYE B.S., MARCHAND B. 1984: Étude ultrastructurale des stades de développement et de la mitose sporogonique de Nosema henosepilachnae n. sp. (Microsporida, Nosematidae) parasite de Henosepilachna elaterii (Rossi, 1794) (Coleoptera, Coccinellidae). Protistologica 20: $165-179$.

VÁVRA J. 1976: Development of the Microsporidia. In: L.A. Bulla and T.C. Cheng (Eds.), Comparative Pathobiology. Vol. 1. Biology of the Microsporidia. Plenum Press, New York, pp. 86-109.

VIVARĖS C.P., SPRAGUE V. 1979: The fine structure of Ameson pulvis (Microspora, Microsporida) and its implication regarding classification and chromosome cycle. J. Invertebr. Pathol. 33: 40-52.

Accepted 22 March 2005 